\title{
Effects of vertical vibration on surface intruder loading in a multiple-size granular system
}

\author{
Wenqing Hou ${ }^{1}$, Shiping Zhang ${ }^{1}$, Ping $W u^{1^{*}}, L_{i} L^{1}$ and Li Wang ${ }^{2 *}$ \\ ${ }^{1}$ School of Mathematics and Physics, University of Science and Technology Beijing, 100083 Beijing, China \\ ${ }^{2}$ School of Energy and Environmental Engineering, University of Science and Technology Beijing, 100083 Beijing, China
}

\begin{abstract}
We investigate the behaviors of the large porous-alumina particles on the free surface of the small glass-grain system under vertical vibration. The experiments are performed using cylindrical container with diameter $\Phi=240 \mathrm{~mm}$, loaded with small glass beads to a static depth $h=100 \mathrm{~mm}$. We control the shaker to vibrate at the various frequencies $f$, from 40 to $80 \mathrm{~Hz}$, and dimensionless acceleration $\Gamma$ $=\left(4 \pi^{2} A f^{2}\right) / g$ from 2 to 6 . When the glass granular system is at rest, porous alumina particles are placed on its free surface. A parameter critical frequency $f_{u}$ is defined to distinguish two kinds of diffusion in particles. When the vibration frequency is less than $f_{u}$, quasi-two-dimensional surface diffusion can occur in porous alumina particles, surface granules clusters formed under certain condition. The frequency and dimensionless acceleration of the vibration are varied to view their effect on the clustering and surface particle-distribution. When the vibration frequency is larger than $f_{u}$, the surface diffusion disappears and a three-dimensional diffusion appears.
\end{abstract}

\section{Introduction}

Surface movement of a vertically vibrating granular system has many unique characteristics. When subjected to vertical vibration, granular systems show many interesting behaviors including pattern formation[1-3], surface fluidization[4-6], convection rolls[7-11], particle separation[12-15], spontaneous heap formation[16-20] and energy dissipation[21]. Detail experiments have classified that surface particles in vibrating system have obvious spontaneous collective effect performance for regularly pattern which is far away from chaotic distribution[16-18]. The investigation of microscopic mechanisms about collective behavior have been done by many authors[22]. In the present study we focused attention on the multiple-size granular system, and discovered a special cluster phenomenon in two-dimensional (2D) multiple-size particle system of a vertically vibration and carried on research on collective cells in different situation.

\section{Experiment}

\subsection{Experiment Setting}

We paid attention on the behavior of the large porous-alumina particles on the free surface of the small glass-grain system under vertical vibration. We used porous activated alumina particles as upper granules, which is a kind of spherical multihole desiccating agent comprised of activated alumina powder. The sine vertical vibration is usually represented as:

$$
z=A \sin (2 \pi f t)
$$

where $A$ stands for vibration amplitude, $f$ is the frequency of vibration.

Surface effects were concerned during the experiment. Walking behavior of these porous alumina particles under vertical vibration was observed through a camera. The important parameters in this experiment are: (i) the diameter of substratum glass particles $\Phi_{l}$ and the diameter of porous alumina particles $\Phi_{2}$. Two kinds of glass grains were used, $\Phi_{l}=1.25 \mathrm{~mm}(1.0-1.5 \mathrm{~mm})$ named as $\mathrm{g}_{1}$ and $\Phi_{1}=0.9 \mathrm{~mm}(0.8-1.0 \mathrm{~mm})$ called $\mathrm{g}_{2}$. Besides, two distinct size alumina granular was $\mathrm{a}_{1}$ with diameter $\Phi_{2}=4 \mathrm{~mm}$ and $\mathrm{a}_{2}$ with diameter $\Phi_{2}=8 \mathrm{~mm}$; (ii) the vibrating parameters: the frequency of vibration $f$ and dimensionless acceleration $\Gamma$ :

$$
\Gamma=\frac{4 \pi^{2} A f^{2}}{g}
$$

where $g$ is the acceleration due to gravity. Moreover, there were other conditions which could impact on the results during the experiment. For example, the shape of the container, the height of glass particle layers $h$ at rest and so on. To curtail the extra effect, we used a cylindrical container which the internal diameter was $\Phi=240 \mathrm{~mm}$ and the static height of glass layer $h$ was always $100 \mathrm{~mm}$. Besides, the same glass under layer particle were used during the experiment. We poured $570 \pm 25(15 \mathrm{~g})$ alumina particles each time.

\footnotetext{
*Ping Wu: pingwu@sas.ustb.edu.cn; Li Wang: liwang@me.ustb.edu.cn
} 


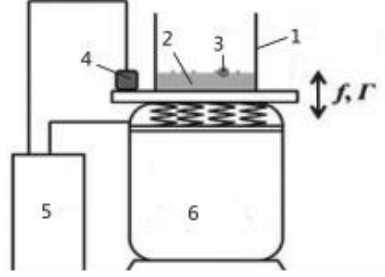

Fig. 1. Schematic of the experimental settings: 1) plexiglass container, 2)glass granular, 3) large porous alumina particles, 4) horizontal balance detector, 5) vibration controlling systems through PC, 6) vibrator apparatus

\subsection{Experiment Results}

\subsubsection{Spontaneous cluster}

In the experiment upper single-layer alumina particles $a_{1}$ were poured into the surface of statically vibrated system which was consisted of small glass particles $\mathrm{g}_{1}\left(\Phi_{1}\right.$ $\left.=1.25 \mathrm{~mm}, \quad \Phi_{2}=4 \mathrm{~mm}, \quad f=30 \mathrm{~Hz}, \quad \Gamma=4\right)$. The alumina particles $a_{1}$ moved radially under the influence of surface wave. We encountered a phenomena in which there were some clusters of particles accompanied by the radial movement in surface behaviors, as shown in Fig. 2.

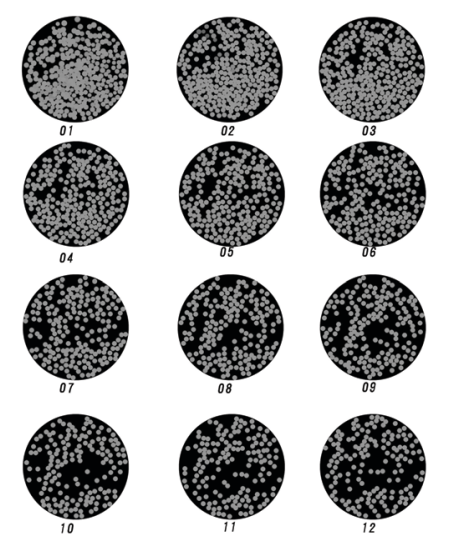

Fig.2. Time-series of the upper alumina particles $a_{1}($ red $)$ surface behavior for under layers glass beads $\mathrm{g}_{1}$ (black) at $\Gamma=4$, $f=30.0 \mathrm{~Hz}\left(\Phi_{1}=1.25 \mathrm{~mm}, \Phi_{2}=4 \mathrm{~mm}\right)$. The time interval was $0.5 \mathrm{~s}$.

Particles collided and merged with one another, forming a particle cluster. The smallest clusters were consisted of two particles. When smallest cluster collided with another particle, the cluster was able to merge with it and "grew up". The life span of cluster cell could be several seconds. The evenly distribution of surface particles had been shattered and alumina particles layer disintegrate into several parts swiftly. However, the general trend of these clusters and particles was radial motion, particles touched the wall of the container and "sank" in glass grains in the end. When cluster phenomenon occurred in alumina particles, they divided into several cells. The dimension of those cells could be different in various conditions $\left(f, \Gamma, \Phi_{1}, \Phi_{2}\right)$, we needed to define a parameter to describe those clusters under same condition. The mean number of particles in one cluster cell under same condition $\left(f, \Gamma, \Phi_{1}, \Phi_{2}\right)$ was defined as $n$. We also defined a parameter $p$ which represented the probability of particles involved in a cluster. Each $(n, p)$ parameters combination show a surface conformation of grains system.

To study the relationship between $(n, p)$ and vibration parameters $(f, \Gamma)$, a camera was used to record the surface conformation change and we counted the grains to keep statistic, as shown in Fig.3 and Fig.4. The unified parameter $\varepsilon$, which contains $\Gamma$ and $t$, marks the activation energy that work on the alumina particle:

$$
\varepsilon=\Gamma \cdot t
$$

where $\Gamma$ is the dimensionless acceleration and $t$ is the time that recorded. We studied the relationship between $(n, p)$ and vibration parameters $(f, \Gamma)$ in cluster phenomenon clearly situation, for example, when $f=40-80 \mathrm{~Hz}$, alumina particles $\mathrm{a}_{1}$ tiled on glass grains $\mathrm{g}_{1}$. Captured the pictures when $\varepsilon=12$ and plotted the statics results as shown in Fig. 3 and Fig. 4.

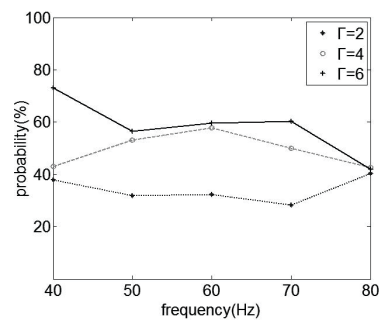

(a)

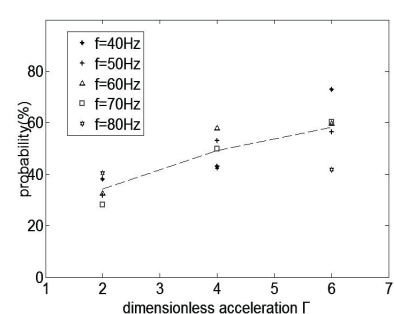

(b)
Fig.3. Diagram for surface alumina grains $a_{1}$ cluster probability $(p)$ and vibration parameter $(f, \Gamma)$ on glass grain $\mathrm{g}_{1}, \varepsilon=12, \Phi_{1}$ $=1.25 \mathrm{~mm}, \Phi_{2}=4 \mathrm{~mm}$. (a) $p$-f relationship; (b) $p-\Gamma$ relationship.

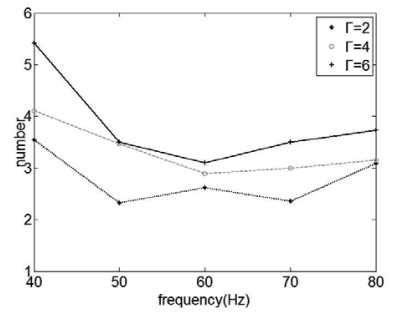

(a)

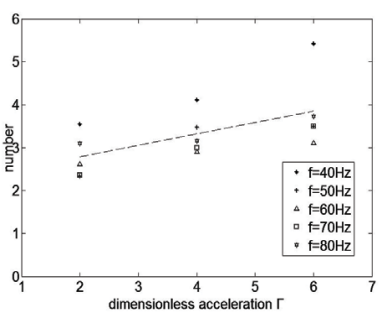

(b)
Fig. 4. Diagram for surface alumina grains $a_{1}$ cluster cell mean particle number $(n)$ and vibration parameter $(f, \Gamma)$ on glass grain $\mathrm{g}_{1}, \quad \varepsilon=12, \Phi_{1}=0.9 \mathrm{~mm}, \Phi_{2}=4 \mathrm{~mm}$. (a) $n-f$ relationship; (b) $n-\Gamma$ relationship.

According to Fig.3 and Fig.4, both two vibration parameters have effects on the scale and the forming probability of cluster cells. However, we could not find brief descriptions of these influences. As cluster phenomenon occurred, surface particles trended to form big cells when $\Gamma$ was smaller, nonetheless, the probability of cluster is lower. By contrast, small cells (cells consisted by $2 \sim 3$ particles) are more likely to form when $\Gamma$ is quiet big, meanwhile, the particles were 
more likely to move individually. What could be said is gamma effected the change more apparent, perhaps, because gamma is directly proportional to short-range repulsive force. Higher frequency probably could only offer more opportunity for particles to recombine clusters, which maybe not play the main part in the time-averaged situation. Dynamics model building is still in progress.

\subsubsection{Multiple-size particles spontaneous cluster}

When the alumina particles were multiple-size, we could observe the spontaneous cluster effect more obviously, see Fig.5. The insertion of larger alumina particles accelerated the formation of cluster cells. Large particles were rapidly packed around by large grains which are close-packed.

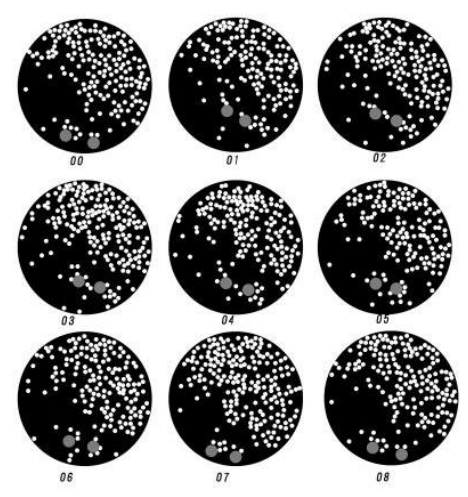

Fig. 5. Time-series of the multiple-size alumina particles $\left(a_{1}\right.$ red and $\mathrm{a}_{2}$ blue) surface behavior for under layers glass bead $\mathrm{s}\left(\mathrm{g}_{1}\right.$ black) at dimensionless acceleration $\Gamma=4, f=30.0 \mathrm{~Hz}\left(\Phi_{1}\right.$ $=1.25 \mathrm{~mm}, \Phi_{2}=4 \mathrm{~mm}$ (red), $8 \mathrm{~mm}$ (blue)). The time interval was $1.0 \mathrm{~s}$.

\subsubsection{Three-dimensional diffusion}

A parameter critical frequency $f_{u}$ is defined to distinguish two kinds of diffusion in particles. When the vibration frequency was bigger than $f_{u}$, the surface diffusion disappeared and a three-dimensional diffusion appeared, as shown in Fig.6. Upper alumina particles sank in the center of glass grains surface, compared with intense convection roll[7] in glass-layer. However, when 2D diffusion appeared, alumina particles only descended when they touch the wall of the container. Besides, the experimental results shown that the critical frequency $f_{u}$ is related to the size and density of particles, but irrelevant with the vibrating intensity parameter $\Gamma$. Granular segregation was first reported in 1939 by Brown[26]. Under certain vertical vibration conditions, large particles in bi-disperse granular mixtures will move to the top of the bed, which is the so-called Brazil nut effect (BNE). Conversely, under certain different vibration conditions, large particles can sink into the granular bed, which is the so-called reverse Brazil nut effect (RBNE)[23]. Kong had indicated that frequency is the decisive factor of such kind of convert between these two effects[22]. An explanation of this phenomenon was proposed in previous studies: as vibration frequency increases, the granular bed is less compact than before, so that big particles sink into the assembly. Similar phenomenon had been observed in our experimental, however, $f_{u}$ is too large for our instrument to work steadily. We didn't careful researches about "sink" phenomenon.

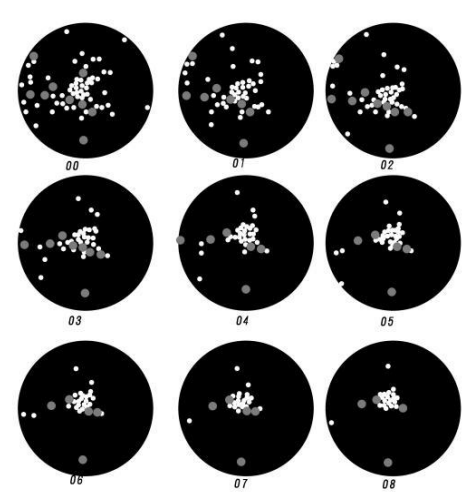

Fig. 6 Time-series of the alumina particles ( $a_{1}$ red and $a_{2}$ blue) 3D diffusion on glass grains $\mathrm{a}_{1}$, at dimensionless acceleration $\Gamma=4, f=100.0 \mathrm{~Hz}\left(\Phi_{1}=1.25 \mathrm{~mm}, \Phi_{2}=4 \mathrm{~mm}\right)$. The time interval was $1.0 \mathrm{~s}$. The colored grains "disappeared" in the center because they descended to the glass layer.

\section{Discussion}

Based on Fig. 3 and Fig.4, both two vibration parameters have effects on the scale and the forming probability of cluster cells. According to the statistic results, we found that although identical particles random walked on the glass beads under the condition of $f<f_{u}$, there was a great chance of surface grains cluster phenomenon instead of even distribution.

When comes to pursuing particle dynamics, the collective motion of granular materials can be described by the excluded volume effect and the dissipation of kinetic energy between granules [19]. A simulation model is described below. A short-range repulsive force was defined as interacting between two particles: the excluded volume is expressed by a short-range repulsive force, which is directly proportional to $\Gamma$ in our experiments, interacting between particles[24]:

$$
f_{i j}=\frac{-\partial \Phi_{i j}}{r_{i j}}
$$

where $\Phi_{i j}$ takes the form of a discrete short-range repulsive pair potential:

$$
\Phi_{i j}=\left\{\begin{array}{l}
\left\{\varepsilon\left[\left(\frac{d}{r_{i j}}\right)^{12}-\left(\frac{d}{r_{i j}}\right)^{6}+\frac{1}{4}\right], i f|r i j|<r_{0}\right. \\
\mathbf{0}, \text { orherwise }
\end{array}\right.
$$

where $r_{i j}=r_{j}-r_{i}$ and $r_{0}=2^{\frac{1}{6}} d, d$ is the characteristic length representing the particle size. Identical grains have same pair potential function in the initial state, considered that all alumina particles could acquire same 
energy $\varepsilon$ during impacting with the glass substrate once. The energy was stored in this distribution is:

$$
E=\int_{i, j=1}^{n} \Phi_{i j}
$$

When $r_{i j}=d$, two particles tangent to each other, the potential of these particles will reach a maximum value. Our discussion is motivated by the "friendship paradox" that "your friends have more friends than you do"[25]. During the vibration, the original distribution has $N$ alumina particles which impact each other $x_{i}$ times. The mean collision times of the system can be determined as $\bar{x}=\sum x_{i} / n$ and the mean transferred energy is $E_{1}=\sum x_{i} e / n$, where $e$ is the transform-energy unit per collision. Thus, the mean number of particles which impact with others' impact number is $x_{w}=\sum x_{i}^{2} / \sum x_{i}$, which is the weighted average of the impact time of each particle. $E_{2}=\sum x_{i}^{2} e / \sum x_{i}$.

$$
x_{w}=\bar{x}+\frac{\sigma^{2}}{\bar{x}}
$$

where $\sigma^{2}$ is variance of the system. Weighted average is bigger than the mean number of the whole system, that means those alumina particles which has ever impact with other particles has transfer more energy. In the other words, there is some probability that some of grains have less probability to connect with others. Some of grains form cluster cells and reduce the chance of collision. When the short-range repulsive force is larger, the energy transfer will be more efficient. Therefore the difference of connecting probability between particles could be more obvious. From what has been discussed above, we may safely explain some of our experiment results.

\section{Conclusions}

We investigated the behavior of the large porous alumina particles on the free surface of the small glass-grain system under vertical vibration. When frequency was lower than critical frequency $f_{u}$, the large grains walked radially and quasi-two-dimensional surface diffusion occurred. For uniform size alumina grains, spontaneous cluster phenomenon had been found and the statistic properties of cluster cells had been researched. When frequency was higher than critical frequency $f_{u}, 2 \mathrm{D}$ diffusion disappear and 3D convection appear. The alumina grains sank in the center of glass beads surface. Lastly, although not presented here, for experiments in high frequency, we found that the parameter $f_{u}$ was not related with vibration parameters. Besides, it was related to the size and density of particles.

This article was sponsored by National Natural Science Foundation of China (No. 51476009).

\section{References}

1. Istafaul $\mathrm{H}$ A, Meheboob A, AIP Conference Proceedings 1542, 775 (2013)

2. Peter E, Ko van der W, Devaraj van der M, Robert B, and Detlef L, Physics of Fluids 19, 123301 (2007);

3. Eric C, Loic V, Jean R, and Jacques D, Phys. Rev. E 53, 2972 (1996)

4. Stephen W, Jonathan M. H, and George T. H. J, Phys. Rev. E 52, 5583 (1995)

5. Thorsten P, Thomas S and Clara S, Phys. Rev. E 62,1361(2000)

6. Vincent M, Lydéric B, and Annie C, Soft Matter 10, 6984-6989 (2014)

7. Frank R, AIP Conference Proceedings 1542, 771 (2013)

8. Frank R and Ralf S, AIP Conference Proceedings 1145, 741 (2009);

9. Sakon K, Charles S. Campbell, Physics of Fluids 20, 103303 (2008);

10. Zhang F, Wang L, Liu C, Wu P, Zhan S, Physics Letters A 378, 1303-1308 (2014)

11. Hu K W, Xie Z A, Wu P, Sun J, Li L, Jia C, Zhang S P, Liu C P, Wang L, Soft Matter 10, 4348-4359 (2014)

12. Xie Z-A, Wu P, Zhang S P, Chen S, Jia C, Liu C P, Wang L, Physical Review E 85, 061302 (2012)

13. Xie Z-A, Wu P, Yang W, Zhao J, Zhang S, Li L, Chen S, Jia C, Liu C, Wang L, Powder Technol. 260, 1-6 (2014)

14. Sun J, Liu C, Wu P, Xie Z-A, Hu K, Wang L, Phy. Rev. E 94, 032906 (2016)

15. Liu C P, Wang L, Wu P, Jia M, Physical Review Letters 104, 188001 (2010)

16. Gauri R. P, Sagar A. Pa, Anil D. G, V. Sitaramamb, Physica A 270, 288-294 (1999)

17. Krishna K, Eric F , Kapil M S, Physica A 270 , 97-104 (1999)

18. Nicholas C, Tom De W, Kristof V, Ilse C, Applied and Environmental microbiology 73, 1809-1824 (2007)

19. Keiko M Ai, Tetsuo A, Phys. Rev. Lett. 77, 4166 (1996)

20. Xie Z-A, Wu P, Wang S, Huang Y M, Zhang S P, Chen S, Jia C, Liu C P, Wang L, Soft Matter 25, 35-57 (2013)

21. Li L, Wu P, Rehman A, Wang L, Zhang S, Xie Z-A, Powder Technol. 307, 84-89 (2017)

22. Xiang-Z K, Mao-B H, Qing-S W, Phys. Lett. A 356, 267-271 (2006)

23. Shinbrot T, Muzzio F J, Phys. Rev. Lett. 81, 4365-4368 (1998)

24. Keiko M. Aoki ,Tetsuo Akiyama, Phys. Rev. E 52, 3288 (1995)

25. Scott L. Feld, The American Journal of Sociology, 96,1464-1477(1991)

26. Brown R.L, Inst. Fuel 13, 15(1939) 